\title{
Dual-layer transposon repression in heads of Drosophila melanogaster
}

\author{
MARIUS VAN DEN BEEK, ${ }^{1,4}$ BRUNO DA SILVA, ${ }^{1}$ JULIETTE POUCH, ${ }^{2}$ \\ MOHAMMED EL AMINE ALI CHAOUCHE, ${ }^{2}$ CLÉMENT CARRÉ, $^{1}$ and CHRISTOPHE ANTONIEWSKII,3 \\ ${ }^{1}$ Drosophila Genetics and Epigenetics; Sorbonne Université, CNRS, Biologie du développement - Institut de Biologie Paris Seine, \\ 75005 Paris, France \\ ${ }^{2}$ Genomic facility, Institut de biologie de I'Ecole normale supérieure (IBENS), Ecole normale supérieure, CNRS, INSERM, PSL Université Paris, \\ 75005 Paris, France \\ ${ }^{3}$ ARTbio Bioinformatics Analysis Facility, Sorbonne Université, CNRS, Institut de Biologie Paris Seine, 75005 Paris, France
}

\begin{abstract}
piRNA-mediated repression of transposable elements (TE) in the germline limits the accumulation of mutations caused by their transposition. It is not clear whether the piRNA pathway plays a role in adult, nongonadal tissues in Drosophila melanogaster. To address this question, we analyzed the small RNA content of adult Drosophila melanogaster heads. We found that the varying amount of piRNA-sized, ping-pong positive molecules in heads correlates with contamination by gonadal tissue during RNA extraction, suggesting that most of the piRNAs detected in heads originate from gonads. We next sequenced the heads of wild-type and piwi mutants to address whether piwi loss of function would affect the low amount of piRNA-sized, ping-pong negative molecules that are still detected in heads hand-checked to avoid gonadal contamination. We find that loss of piwi does not significantly affect these 24-28 nt RNAs. Instead, we observe increased siRNA levels against the majority of Drosophila TE families. To determine the effect of this siRNA level change on transposon expression, we sequenced the transcriptome of wild-type, piwi, dicer-2 and piwi, dicer-2 double-mutant heads. We find that RNA expression levels of the majority of TE in piwi or dicer-2 mutants remain unchanged and that TE transcripts increase only in piwi, dicer-2 double-mutants. These results lead us to suggest a dual-layer model for TE repression in adult somatic tissues. Piwi-mediated gene silencing established during embryogenesis constitutes the first layer of TE repression whereas Dicer2-dependent siRNA-mediated silencing provides a backup mechanism to repress TEs that escape silencing by Piwi.
\end{abstract}

Keywords: Dicer; piwi; transposable elements; piRNAs; siRNA

\section{INTRODUCTION}

Transposable element (TEs) activity is thought to be an important force in genome evolution, as TE integration and excision can result in mutations that impact gene regulation networks (Tubio et al. 2014). However, these mutations may be detrimental to individuals, potentially decreasing lifespan and fertility (Wood et al. 2016). Therefore, limited TE mobilization is beneficial to both host and TE, whereas high TE activity decreases host fitness and adversely affects vertical transfer of the TE.

In Drosophila melanogaster, the siRNA (small-interfering RNA) and the piRNA (piwi-interacting RNA) pathways

\footnotetext{
${ }^{4}$ Present address: Institut Curie, PSL Research University, CNRS UMR 3215, INSERM U934, Stem Cells and Tissue Homeostasis, 75005 Paris, France

Corresponding authors: Christophe.antoniewski@sorbonneuniversite.fr, clement.carre@sorbonne-universite.fr

Article is online at http://www.rnajournal.org/cgi/doi/10.1261/rna. 067173.118. Freely available online through the RNA Open Access option.
}

are important negative regulators of TE expression in somatic (Ghildiyal et al. 2008; Li et al. 2013) and gonadal tissues (Vagin et al. 2006), respectively. Both pathways are active in the gonads, while the siRNA pathway is thought to be active in all somatic tissues. Dicer-2, the central endonuclease of the siRNA pathway, operates on double-stranded RNA (dsRNA) molecules by processively translocating along the molecule and cutting every $21 \mathrm{st}$ nucleotide (Cenik et al. 2011; Welker et al. 2011). After the initial processing, these 21 nt duplexes with $3^{\prime} \mathrm{OH}$ overhangs of $2 \mathrm{nt}$ are rebound by Dicer- 2 and $\mathrm{r} 2 \mathrm{~d} 2$ to be loaded into Argonaute-2 (Ago2). Ago2 can then engage in multiple rounds of endonucleolytic cleavage of transcripts with mature siRNA complementarity. Loss of the functional siRNA pathway results in increased levels of TE expression and mobilization (Czech et al. 2008; Ghildiyal et al. 2008;

(C) 2018 van den Beek et al. This article, published in RNA, is available under a Creative Commons License (Attribution 4.0 International), as described at http://creativecommons.org/licenses/by/4.0/. 
Li et al. 2013; Xie et al. 2013) and compromised male fertility as well as defective sperm development (Wen et al. 2015).

The Drosophila piRNA pathway is well characterized for its role in maintaining germline stem cells (Cox et al. 1998; Jin et al. 2013) and genome integrity in the gonads and early embryo (Wang and Elgin 2011), and is therefore required for fertility. Its key components are three germline Argonaute-family proteins: Piwi, Aubergine (Aub), and Argonaute-3 (Ago3). piRNA production relies on the processing of piRNA cluster transcripts, which are composed of TE fragments. The processing of these primary transcripts by the piRNA biogenesis machinery produces piRNAs which are loaded into Piwi or Aub in germline cells' cytoplasmic structure called the nuage (Brennecke et al. 2007). Aubergine loaded with antisense piRNAs can thus slice complementary sense TE transcripts. The phosphodiester bond cleavage event on the targeted TE transcript occurs opposite to the nucleotides 10th and 11th of the antisense piRNA, thus becoming the $5^{\prime}$ end of a new TE sense piRNA. Sense piRNAs are then loaded onto Ago3 and trimmed, which in turn cleaves complementary antisense piRNA cluster transcripts opposite to the position 10th and 11th of the piRNA. This cytoplasmic cyclic process referred to as secondary piRNA amplification leaves a detectable "ping-pong" signature, in which 5'-piRNA ends tend to overlap by 10 nucleotides (Brennecke et al. 2007; Gunawardane et al. 2007).

piRNA-loaded Piwi proteins can reenter the nucleus, where piRNAs guide Piwi toward complementary nascent transcripts. Piwi then recruits factors (Maelstrom, SuVar3-9, dSETDB1, HP1, and silencio/Panoramix) that establish and maintain $\mathrm{H} 3 \mathrm{~K} 9 \mathrm{me} 3$ at the surrounding genomic vicinity of TE insertion sites, possibly including protein coding genes and hence functions in TGS (Brower-Toland et al. 2007; Rangan et al. 2011; Wang and Elgin 2011; Sienski et al. 2012, 2015).

Zygotic piwi expression has been detected ubiquitously in early embryos up to the 14th nuclear division ( $~ 2 \mathrm{~h}$ after egg laying) (Rouget et al. 2010; Mani et al. 2014), depletion of Piwi in nurse cells and oocytes results in early arrest of embryonic development (Wang and Elgin 2011; Mani et al. 2014), and piwi acts as a suppressor of variegation in the eye (Pal-Bhadra et al. 2004; Gu and Elgin 2013), suggesting an important function for Piwi in germline cells' maintenance and during early development of somatic tissues. In contrast, the function of Piwi in larval or adult somatic tissue remains unclear. Piwi has both been reported to be present (Brower-Toland et al. 2007) or absent (Le Thomas et al. 2013) in third instar larval salivary glands, whereas Aubergine and Ago3 have been observed in nonoverlapping cells of the adult central nervous system (Perrat et al. 2013).

In order to unravel the role of the piRNA pathway in TE control of somatic adult tissues, we analyzed small RNA profile in wild-type, piwi and dicer-2 mutant fly heads.
We provide evidence that previously reported ping-pong pairs in adult heads likely result from contamination with testicular RNA, suggesting that secondary piRNA amplification does not take place in adult heads. However, small RNA sequencing of piwi mutant heads reveals an increased level of siRNAs against most TE families. RNA-sequencing of piwi single mutant heads and dicer-2 single mutant heads showed only minor up-regulation of TEs, whereas double-mutants of piwi and dicer-2 showed increased TE levels. Our results suggest a dual-layer model of TE repression in somatic tissues. The first layer of TE repression is established by Piwi at the chromatin level during early development as shown by Gu and Elgin (2013). When TEs escape the epigenetic Piwi silencing, PTGS triggered by dicer-2 and siRNAs mediates TE degradation to decrease TE burden.

\section{RESULTS}

\section{Secondary piRNA biogenesis is not detectable in adult heads}

Ago3 and Aubergine were detected by immunofluorescence in the optic lobe of the Drosophila central nervous system (Perrat et al. 2013), and several teams sequenced small RNA molecules in head samples of wild-type and mutants that had the size (24-28 nt), 3'-end 2'-O-methylation and ping-pong signature of Drosophila piRNAs (Ghildiyal et al. 2008; Yan et al. 2011; Mirkovic-Hösle and Förstemann 2014). With the exception of Ghildiyal and coworkers' data set, which was obtained from Ago2 mutant heads, these putative piRNAs corresponded to a very minor fraction of the sequence data sets, which raises the possibility of sample contamination by gonadal tissues during RNA extraction. To address this issue, we analyzed small RNA matching TE sequences in 24 small RNA sequencing libraries prepared from adult male heads isolated by sieving after freezing (Reinhardt et al. 2012). The majority of reads were in the size range of siRNAs (21 nt). However, we observed a fraction of reads in each library within the 24-28 nt size range of Drosophila piRNAs (mean 7.5\%, interquartile range 5.6\%-10.4\%; Supplemental Fig. 1A,B).

We searched for ping-pong partners among 24-28 nt reads for each of the 24 small RNA sequencing libraries, as had been done in previous works (Ghildiyal et al. 2008; Yan et al. 2011; Mirkovic-Hösle and Förstemann 2014). We detected a significant ping-pong signature ( $>20$ pairs, $z$-score of the 10 nt overlap $\geq 2$ ) in five out of the 24 analyzed libraries (hereafter referred to as "ping-pong positive" libraries, Supplemental Fig. 1C, red asterisks), while seven libraries (hereafter referred to as "ping-pong negative" libraries, Supplemental Fig. 1C, black square) had a negative 10 nt overlap z-score.

The genotype of any of the analyzed libraries was not expected to differentially affect piRNA biogenesis, 
suggesting that the significant signatures we found might be due to stochastic contamination by gonadal material during the RNA extraction from heads prepared from adult male isolated by sieving after freezing (Reinhardt et al. 2012). To investigate this possibility, we performed differential expression testing of miRNAs between the five ping-pong positive libraries and the seven pingpong negative libraries, under the assumption that RNA introduced from contaminating tissues would include tissue-specific miRNAs that are not normally expressed in heads. Under our assumption, those putative tissue-specific miRNAs would be absent in ping-pong negative head libraries (not contaminated) and present in ping-pong positive head libraries (contaminated).

A set of 27 miRNAs was significantly enriched in pingpong positive libraries relative to ping-pong negative libraries at an adjusted $P$-value (Benjamini-Hochberg) of 0.01 (Supplemental Table S1). Since we analyzed male heads, the contaminating RNA giving rise to ping-pong signature might stem from the testis. To investigate this possibility, we randomly added reads from testicular small RNA libraries (Rozhkov et al. 2010; Toledano et al. 2012) to ping-pong negative head libraries whose sizes were normalized by sampling to $2.5 \times 10^{6}$ reads for comparison. We found that adding $\sim 2 \%$ testes reads to mimic the contamination in the ping-pong negative head libraries is sufficient to detect a ping-pong signature ( $>20$ pairs, $z$-score for 10 nt overlap $\geq 2$; Supplemental Fig. 1D).

In addition, we found a strong overlap of miRNAs differentially expressed between ping-pong positive and pingpong negative libraries on the one hand, and miRNAs differentially expressed between ping-pong negative libraries with or without simulated testis contamination on the other hand: The 10 most significantly changed miRNAs between ping-pong negative libraries with or without $2 \%$ of testicular reads are also significantly changed between ping-pong positive and ping-ping negative libraries (nine miRNAs with $P<0.01$, one miRNA with $P<$ 0.025; Supplemental Table S1; Supplemental Fig. 1E). Altogether, these data strongly suggest that when detected in the head, the ping-pong signature is mostly due to contamination by gonadal abundant small RNAs during sample preparation, in particular if a sieving strategy after freezing is used to isolate the heads and not followed by eye check. However, we cannot exclude that Aubergine and Ago 3 are producing secondary piRNAs in very low amounts or in a small set of head cells.

\section{Ping-pong negative piRNA-like small RNAs in adult heads are not piwi-dependent}

In agreement with our previous observations, new libraries prepared from wild-type $(+/+)$, heterozygous ( piwi $+/-$ ), or homozygous (piwi -l-) piwi, and heterozygous (dicer-2 $+/-$ ) or homozygous (dicer-2 -/-) dicer-2 heads carefully checked to avoid contamination by gonadal debris were ping-pong negative (Supplemental Fig. 1F). However, we still detected a small amount of piRNA-sized reads (24-28 nt) in these libraries (Fig. 1A, insets). Since Piwi is necessary for piRNA biogenesis without ping-pong signature in ovarian follicle cells (Lau et al. 2009; Robine et al. 2009; Saito et al. 2009), we further explored the possibility that these 24-28 nt piRNA-sized reads are Piwi-dependent primary piRNAs.

As the main function of Piwi has been proposed to induce transcriptional repression of TEs in ovaries (Sienski et al. 2012; Rozhkov et al. 2013), we focused on small RNA reads aligning to TEs.

We observed that for wild-type, piwi heterozygous and piwi homozygous mutant heads, the 24-28 nt reads show a slight bias for aligning to the sense strand of TEs (Fig. $1 \mathrm{~A}$, inset), which is further amplified in dicer-2 mutant flies. This is in contrast to piRNA molecules in gonads that predominantly align to the antisense strand of TEs (Brennecke et al. 2007). Importantly, the fraction of 24-28 nt antisense to TEs seemed to increase slightly in piwi homozygous mutant heads. Together, these results suggest that the small fraction of 24-28 nt RNAs in ping-pong negative head libraries are not piwi-dependent RNAs (Fig. 1B).

\section{Piwi mutation unmasks a TE-specific siRNA response in adult somatic tissues}

We next examined whether loss of piwi would affect siRNAs that align to TE sequences in the head. In both wild-type and heterozygous piwi mutant heads, we detected a substantial amount of 21-nt sense (wild-type: $16,070 \pm 1099$ counts per 10 millions aligned reads in library [cp10m], piwi heterozygous 18,643 $\pm 4199 \mathrm{cp} 10 \mathrm{~m}$ ) and antisense (wild-type: 17,140 $\pm 1069 \mathrm{cp} 10 \mathrm{~m}$, piwi heterozygous 19,207 $\pm 197 \mathrm{cp} 10 \mathrm{~m}$ ) reads, which increases in homozygous piwi mutant (sense: 29,903 $\pm 6531 \mathrm{cp} 10 \mathrm{~m}$, antisense: $29,117 \pm 5325 \mathrm{cp} 10 \mathrm{~m}$ ) (Fig. 1A,E). Importantly, these reads are strongly reduced in dicer-2 mutants (dcr2G31R sense: $2205 \mathrm{cp} 10 \mathrm{~m}$, dcr2 -/- sense $2130 \mathrm{cp} 10 \mathrm{~m}$, dcr2-G31R antisense: $1339 \mathrm{cp} 10 \mathrm{~m}, \mathrm{dcr} 2$-/- antisense $720 \mathrm{cp} 10 \mathrm{~m})$, confirming that TE-aligned reads are siRNAs (Fig. 1A,E). We consistently observed the increase of 21 nt sense and antisense RNAs in piwi homozygous mutant heads when considering only TE families that had on average more than 20 aligned reads per 10 million matched reads ("cp $10 \mathrm{~m}$ "). To quantify siRNA variations we only considered the fraction of $21 \mathrm{nt}$ reads that aligns to the TE complementary strand, in order to minimize quantification of partially degraded TE transcripts, which would be expected to align to the sense strand with little size specificity (discussed in Malone et al. 2009). We further restricted the analysis to TE families that had on average five or more cp10m of 21-nt antisense reads. By doing so, we determined that in piwi mutants, 27 out of 62 TE families 
A

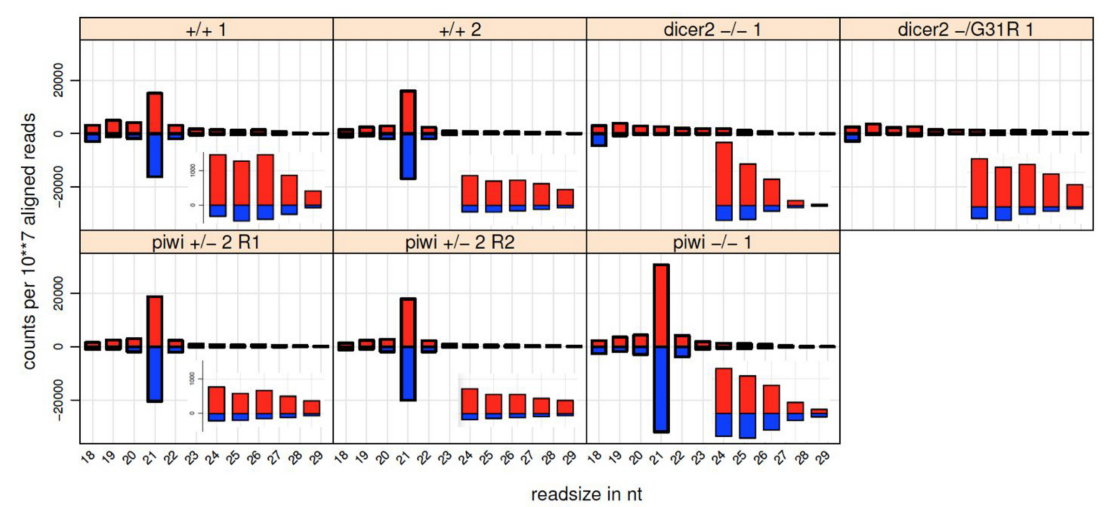

C
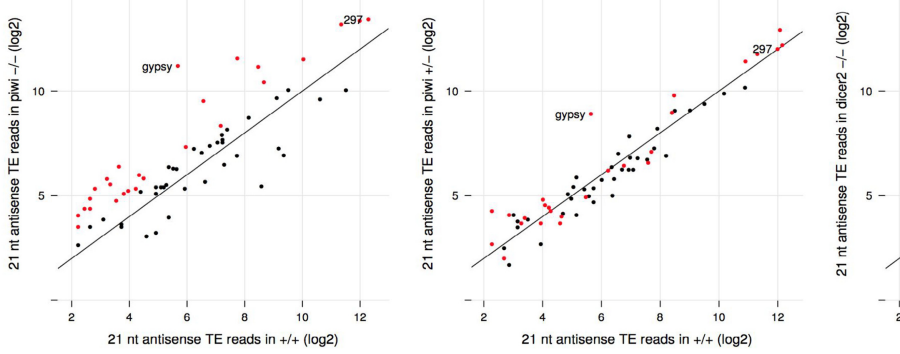

B

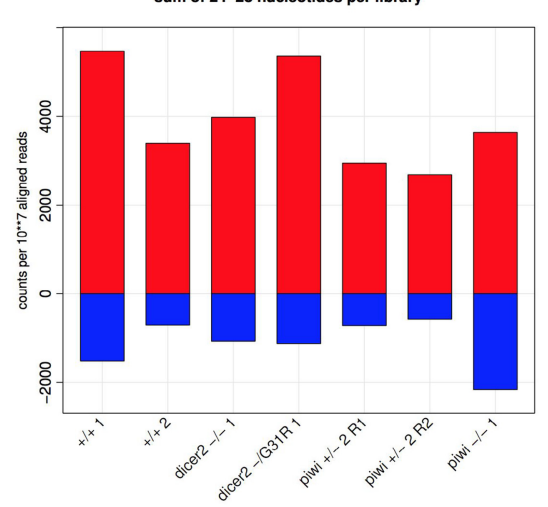

D

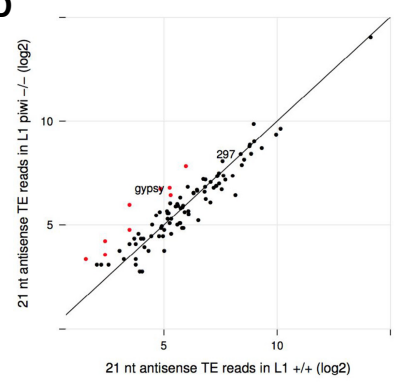

E

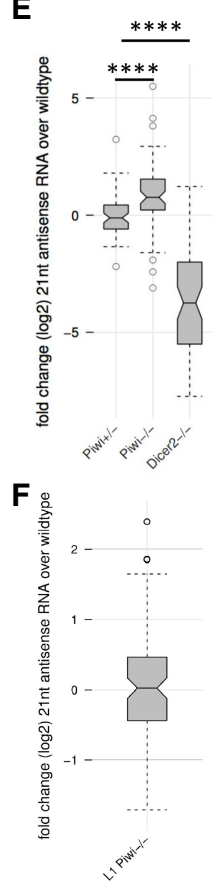

G

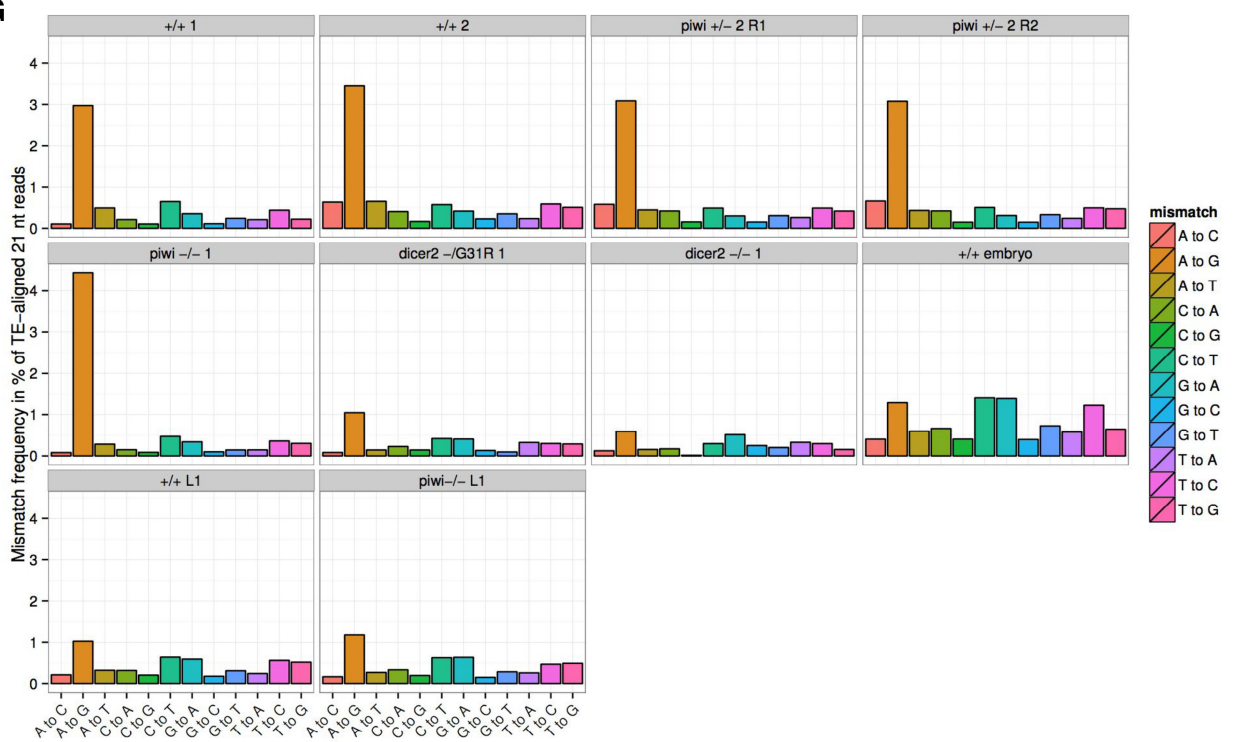

FIGURE 1. Loss of Piwi repression leads to an increase of TE-targeting siRNAs. (A) Overview of the size ( $x$-axis) and amount ( $y$-axis, in counts per 10 million mapped reads) of small RNA reads that align to TEs in adult heads of the indicated genotype. Zoom of the 24-28 nt fractions are shown as insets. (B) Sense or antisense reads of 24 to $28 \mathrm{nt}$ were summed for each indicated genotype ( $x$-axis). (C) Scatterplots displaying the abundance of $21 \mathrm{nt}$ antisense reads in mutant ( $y$-axis) and wild-type ( $x$-axis) heads. Red dots in the first panel indicate the transposon-specific $21 \mathrm{nt}$ antisense reads that increased more than twofold in piwi homozygous mutant heads. These dots are shown for comparison in the second and third panels. (D) same as $C$ but for wild-type first instar larvae (homozygous piwi mutant versus wild-type). (E) Boxplots showing the distribution of $21 \mathrm{nt}$ antisense fold changes ( $y$-axis) between wild-type and the indicated mutants ( $x$-axis). Significance of differences between the distributions was assessed with Mann-Whitney U-test. (F) As in $E$, but for wild-type first instar larvae (homozygous piwi mutant versus wild-type). (G) Mismatches of $21 \mathrm{nt}$ reads aligning to reference genome TE insertions with one mismatch allowed. Identity of mismatch is indicated on the $x$-axis, and the fraction of all reads with this mismatch identity over all TE matches is indicated on the $y$-axis. In all panels, the index of the sample duplicate is indicated after the genotype when appropriate. 
show greater than twofold increases of siRNAs, with an overall median fold change of 1.69 compared to a 0.92 fold change for piwi heterozygous mutant heads (Fig. 1C, P-value $1.03 \times 10^{-05}$, Mann-Whitney $U$ ). While most TE siRNAs were hence moderately increased, gypsy siRNA expression was increased more than 45-fold.

We also investigated if the observed changes would occur at earlier time points during development. We observed a 2.8-fold increase of gypsy-specific siRNA in piwi $^{-1-}$ mutant first instar larvae (L1) as compared to piwi $^{+/+} \mathrm{L1}$; however, the majority of TE family siRNAs remained unchanged at this stage ( $P$-value 0.83 , MannWhitney U, Fig. 1D). In a previous work (Pal-Bhadra et al. 2004; Gu and Elgin 2013), the knockdown of piwi in the early embryo but not in larvae suppressed the silencing of $w^{m 4}$ in adult eyes, suggesting that a role of Piwi in establishing the repressive chromatin state of $w^{\mathrm{m} 4}$ is fulfilled during early embryogenesis. Our observation that the zygotic piwi loss of function has a stronger effect on TE siRNA levels in adults than in larvae is in agreement with the view of a TE repression established through a maternally inherited function of piwi that would be gradually lost during development in zygotic piwi mutants. However, we cannot exclude that increased TE siRNA levels in piwi adult mutant heads rather reflects a late zygotic function of piwi in these tissues, which remains to be demonstrated.

\section{The siRNA response likely originates from Dicer-2- mediated TE transcript processing in adult heads}

We next investigated the origin of the increase in TE derived siRNAs in piwi mutant heads. siRNA response might be a direct consequence of increased TE transcription in the absence of Piwi, which in turn would result in increased processing of TE-derived dsRNAs by Dicer-2 into siRNAs. Alternatively, the elevated levels of TE-siRNAs might originate from piRNA clusters, which produce both piRNAs and siRNAs in the germline. If this were to be the case, we should be able to detect an increase of specific 21-nt RNAs originating from these piRNA clusters.

As most germinal piRNA clusters are transcribed bidirectionally, we quantified both 21 nt sense and antisense RNAs that map exclusively to piRNA clusters (defined in Brennecke et al. 2007). We thereby excluded sequences shared with TE insertions elsewhere in the genome, allowing us to separate production of siRNAs originating from clusters and those originating from TE insertions. We observed a low quantity of $21 \mathrm{nt}$ reads derived from piRNA cluster in both piwi mutant and control wildtype heads (between $2 \%$ and $3.5 \%$ of total TE reads, Supplemental Fig. 2A). These cluster-derived $21 \mathrm{nt}$ RNAs increased 1.44-fold in piwi mutant heads, which is less than the 2.15-fold increase of $21 \mathrm{nt}$ antisense TE reads (Supplemental Fig. 2B). The increase of TE-specific siRNAs in piwi mutant heads was thus unlikely to be caused by an increase in Dicer-2-mediated processing of piRNA cluster transcripts, favoring the hypothesis in which increased transcription of euchromatic TE insertions leads to an increase in TE-specific siRNA production through Dicer-2 activity. In principle, the increase of TE-specific siRNAs could be maternally inherited or stably maintained from early development. We took advantage of the fact that mature Ago2-loaded siRNAs are single-stranded and that double-stranded RNAs, among which are the substrates of Dicer-2, are frequently deaminated through the action of adenosine deaminase acting on RNA (ADAR) enzyme, which converts adenosine (A) to inosine (I) (Palladino et al. 2000; Keegan et al. 2005; Wu et al. 2011). This change manifests in $A$ to $G$ mismatches in RNA sequencing data sets as compared to the DNA based reference genome. We therefore determined the frequency of all nucleotide mismatches for all FlyBase-listed TE insertions in our small RNA sequencing data sets (Fig. 1G). The amount of $A$ to $G$ mismatches is not elevated over other mismatches in early embryos (1.5\% of all $21 \mathrm{nt}$ reads matching to TE insertions). We detect a similar frequency of $A$ to $G$ mismatches (1.2\% to $1.4 \%$ ) in first instar larvae; however, no other mismatches were preferred, suggesting that ADAR might be active at low level in 1 st instar larvae. In contrast, we detected a higher frequency of $A$ to $G$ mismatches in wild-type heads $(3.6 \%-4.1 \%)$ that, despite a greater than twofold increase of $21 \mathrm{nt}$ RNA, further increases in piwi mutants (5.7\%). Since this increase would not be observed if siRNA were inherited from earlier developmental stages, the data suggest that siRNA are actively produced by Dicer-2 from double-stranded TE RNA substrates in piwi mutant adult heads.

\section{Neither loss of Piwi nor Dicer-2 leads to strong up-regulation of TEs}

To determine whether the observed increase of siRNA production efficiently counteracts any increased TEs transcription caused by a lack of Piwi-mediated TGS at an earlier stage, we sequenced the head transcriptome of piwi mutants, dicer-2 mutants and piwi, dicer-2 double-mutants and compared these to wild-type head transcriptome. In piwi mutants, transcript levels of most TEs remain unchanged in those heads, with the notable exception of gypsy, whose level increases about fivefold (Fig. 2A). Gypsy is also the TE against which we observed the strongest increase of siRNA levels (Fig. 1C), suggesting that the transcription of TEs is indeed increased in piwi mutant heads and correlates with Dicer-2 dependent siRNA production through the dicing of double-stranded TE RNAs.

Contrary to sense transposon transcripts, we did detect a significant increase in antisense transcripts in piwi mutant heads (Fig. 2B, +/+ versus Piwi -/- blue boxes), that might form transient duplexes with sense TE transcripts, serving as a substrate for Dicer-2-mediated siRNA processing. 
A

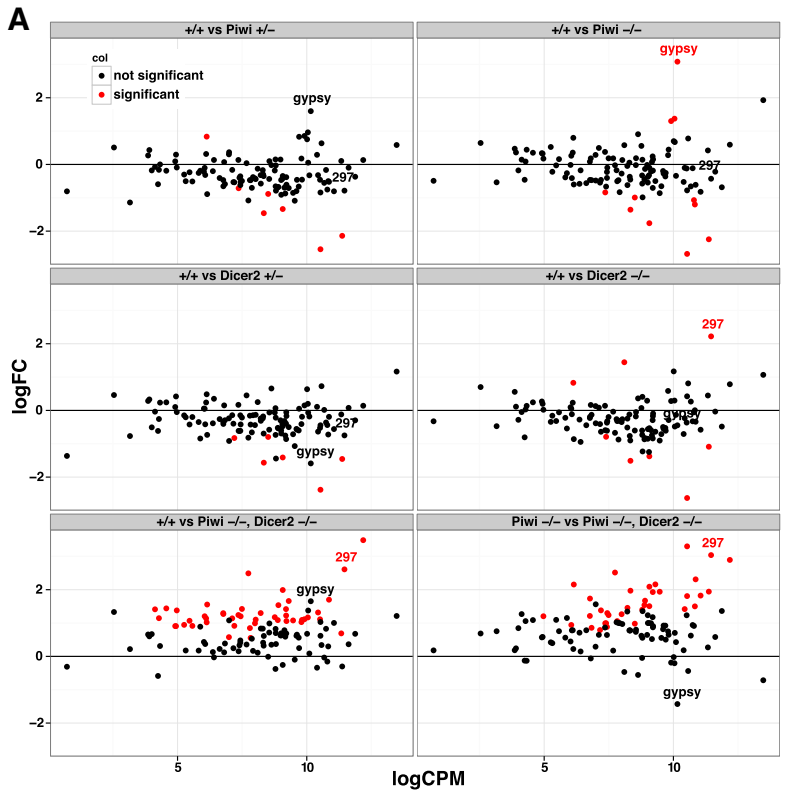

C

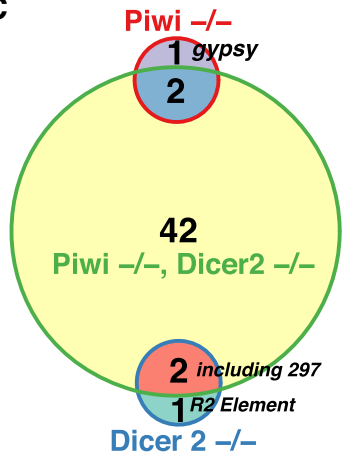

B

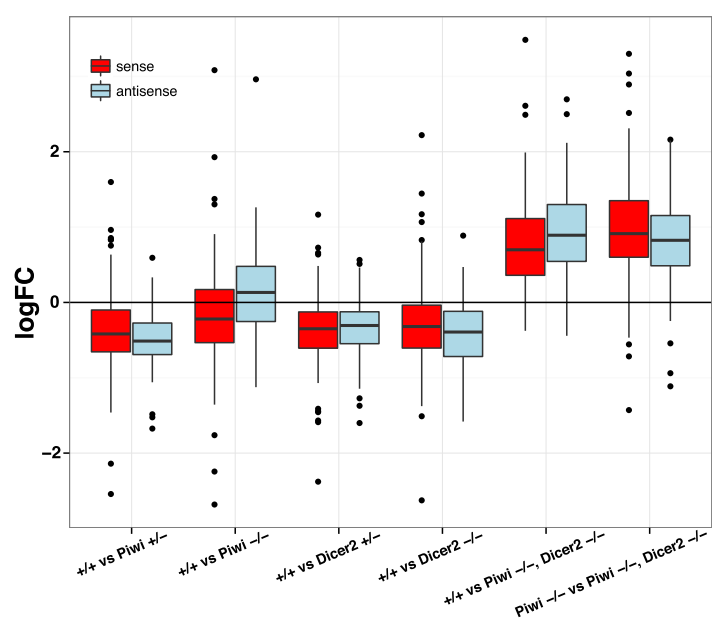

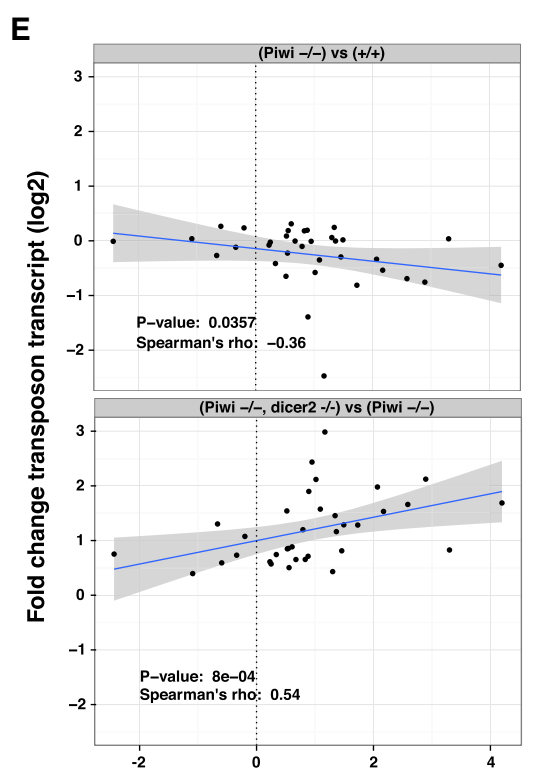

Fold change siRNA in piwi mutant $(\log 2)$

FIGURE 2. Piwi and Dicer-2 are complementary factors for the repression of TEs in adult heads. (A) Scatterplots displaying the log 2 fold changes of sense TE transcript expression on the $y$-axis and the mean expression strength on the $x$-axis. $\log _{2}$ fold changes were calculated between the indicated mutant and the wild-type TE levels, except in the last panel where we calculate changes between the double mutant and the piwi mutant. (B) Boxplots showing the distribution of $\log _{2}$ fold changes as in $A$, but considering changes of sense (red) and antisense TE transcripts (blue) separately for each comparison (x-axis). (C) Venn diagram showing the overlap of significantly (p.adj $<0.05$ ) up-regulated TEs for the indicated mutants as compared to the wild-type control. (D) As in C, but taking TEs whose transcript abundance increases more than $25 \%$ over the wild-type. (E) Scatterplot displaying the correlation between $\log _{2}$ fold changes of $21 \mathrm{nt}$ antisense RNA (siRNA) in piwi homozygous mutant heads compared to wild-type heads on the $x$-axis and $\log _{2}$ fold changes of sense TE transcripts for the genotype comparisons indicated above each panel. Only TEs that passed a threshold of on average five $21 \mathrm{nt}$ antisense reads (after library-wise normalization, see Materials and Methods) over all small RNA libraries were analyzed. Further, only TEs whose sense transcript level increased in piwi, dicer-2 while insensitive to piwi loss are shown. A scatterplot including all tested genotypes that depict all TEs that passed the siRNA threshold can be found in Supplemental Figure $2 \mathrm{~B}$. The blue line is a fit produced by the Imfit function, and the gray area delimits the corresponding confidence interval.

Similarly, most TEs are not up-regulated in dicer-2 mutants, except for 297, which produces a significant amount of siRNA in wild-type heads, perhaps indicating inefficient Piwi-mediated TGS for this TE family.
We conclude that Piwi and Dicer-2 are redundant for the maintenance of TEs repression for most TE families in adult heads and that both Piwi-mediated TGS and Dicer-2-mediated PTGS can efficiently repress TEs. 


\section{Piwi and Dicer-2 compensatory mechanism revealed in double mutant}

To confirm our hypothesis that the siRNA response in adult somatic tissues compensates for the loss of Piwi earlier in development, we analyzed RNA libraries from piwi, dicer-2 double-mutant heads. We found that the majority of TE families is significantly up-regulated in piwi, dicer-2 double-mutant heads compared to single piwi or dicer-2 mutants (Fig. 2A-C).

To illustrate the impact of the increased siRNA levels in piwi mutants on TE transcripts, we plotted for each TE family the $\log _{2}$ of the fold change of siRNAs in piwi mutants on the $x$-axis, and the fold change in TE transcript level on the $y$-axis. We see a tendency for TE transcripts that are targeted by more siRNAs in piwi mutants to decrease in abundance when comparing piwi mutant to the wild-type (Fig. $2 \mathrm{E}$, top panel). If we perform the same analysis but focus on the change of TE levels between piwi mutant and piwi, dicer-2 double mutants (Fig. 2E, bottom panel), we see a tendency for TEs transcripts to increase in abundance in the double mutant. This further suggests that increased siRNA production in piwi mutants efficiently counteracts loss of the transcriptional repression (TGS) established by piwi earlier during embryogenesis. If this compensation is failing in piwi, dicer-2 double mutants TE transcript levels increase globally.

Altogether our results suggest a dual layer expression control of Piwi-mediated TGS and Dicer-2-mediated PTGS to firmly repress TEs levels in the adult soma.

\section{Piwi and Dicer2 do not restrict expression of genes containing TEs in their genomic loci}

Piwi has previously been shown to silence genes adjacent to TEs insertions and genes that carry TEs sequences in their boundaries (Sienski et al. 2012). Our transcriptomic data does not support a significant trend of up-regulation of genes that contain TE insertions in their genomic boundaries, whether we consider all TE families, or only those that are up-regulated in piwi, dicer-2 or piwi, dicer2 double-mutants (Fig. 3A). Piwi has also been shown to repress genes that produce traffic jam class piRNAs from their 3'UTR (Robine et al. 2009). Again, we detect no expression bias for these genes in any of our heads' mutant conditions (Fig. 3C).

\section{DISCUSSION}

\section{Origin of piRNA-like molecules in somatic tissues}

Through the analysis of a large number of small RNA sequencing libraries, we revisited previous reports of putative secondary piRNA biogenesis in adult heads (Ghildiyal et al. 2008; Yan et al. 2011; Mirkovic-Hösle and Förstemann 2014). Based on our analysis, it is likely that earlier observa- tions resulted from small contamination with gonadal RNA, which can easily occur when collecting heads by vortexing frozen flies and filtering fly-parts by sieving. piRNAlike molecules in adult Drosophila heads were previously observed in libraries that were $\beta$-eliminated before sequencing, and/or that originated from siRNA pathway gene mutants (Ghildiyal et al. 2008; Mirkovic-Hösle and Förstemann 2014). $\beta$-elimination prevents sequencing of small RNAs with unprotected $3^{\prime} \mathrm{OH}$ groups, thereby increasing the apparent sequencing frequency of $2^{\prime} \mathrm{O}$ methylated contaminating piRNAs from gonadal origin. Likewise, decrease of siRNA sequencing in siRNA pathway mutant heads is expected to amplify the sequencing frequency of piRNAs, including contaminants from gonadal origin.

Our results highlight the importance of controlling the purity of RNA preparations. Considering the enormous increase of TE expression in piRNA mutant ovaries, often reaching more than 1000-fold up-regulation, best practice for analysis should be a control that clearly shows the degree of tissue purity of the RNA preparation. We show that careful examination and hand-selection of fly heads eliminates most of the artifactual detection of piRNA ping-pong patterns in head RNA preparations.

When contamination is limited, the marginal fraction of the remaining 24-28 nt RNAs is not sensitive to Piwi loss. This implies that either these piRNA-like molecules are primary piRNAs produced independently of Piwi (Nagao et al. 2010) or that they are degradation products. Together with their sense bias, the absence of a marked peak in the piRNA-size range (Fig. 1A) and the low transcriptional activity of piRNA clusters in heads, these observations strongly suggest that these 24-28 nt RNAs are not piRNAs. The absence of piRNAs in nongonal somatic tissues of Drosophila melanogaster is supported by a recent work suggesting that the piRNA pathway was involved in somatic TEs and mRNAs regulation in an ancestral arthropod. This function is still present in many arthropods but was lost during evolution of the melanogaster subgroup of Drosophila (Lewis et al. 2018).

\section{Piwi likely exerts its function in adult heads through inherited transcriptional repression set in early development}

Zygotic piwi mutations are suppressors of variegation, revealing a link between the piRNA pathway and heterochromatin silencing (Pal-Bhadra et al. 2004). In contrast, late inactivation of piwi in eye imaginal discs does not suppress variegation (Gu and Elgin 2013), suggesting that Piwi silencing established during early embryogenesis is maintained in the absence of Piwi product. As we were unable to evidence a role of piRNAs in TE silencing in adult heads and found that zygotic loss of piwi alone has little 

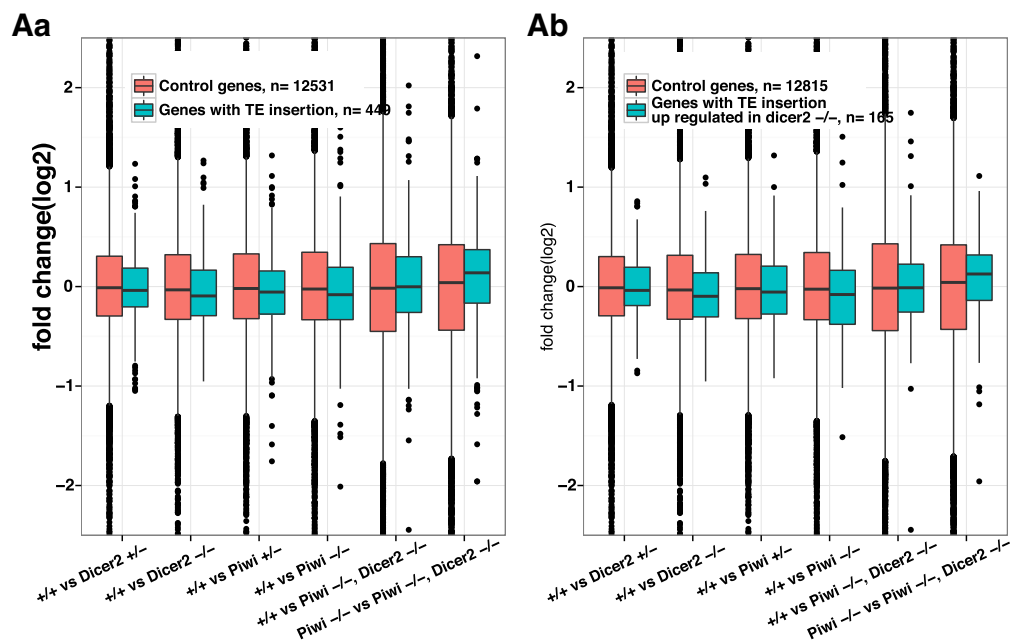
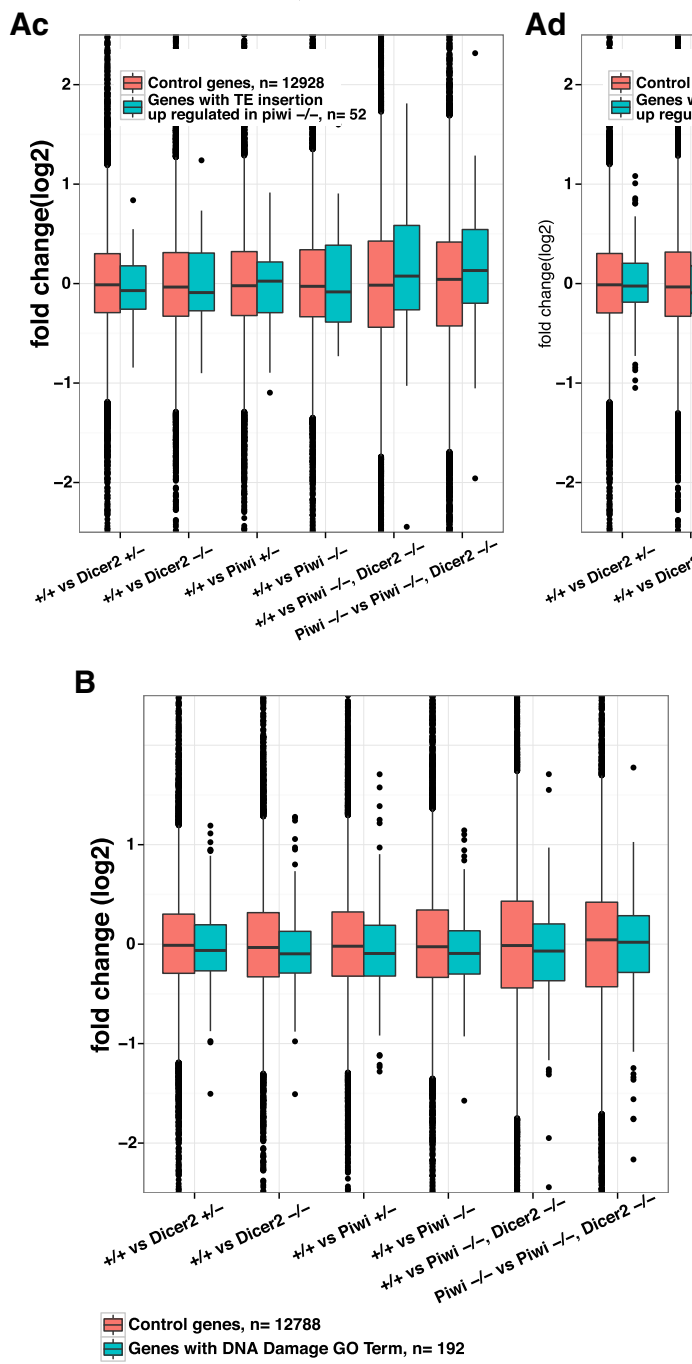
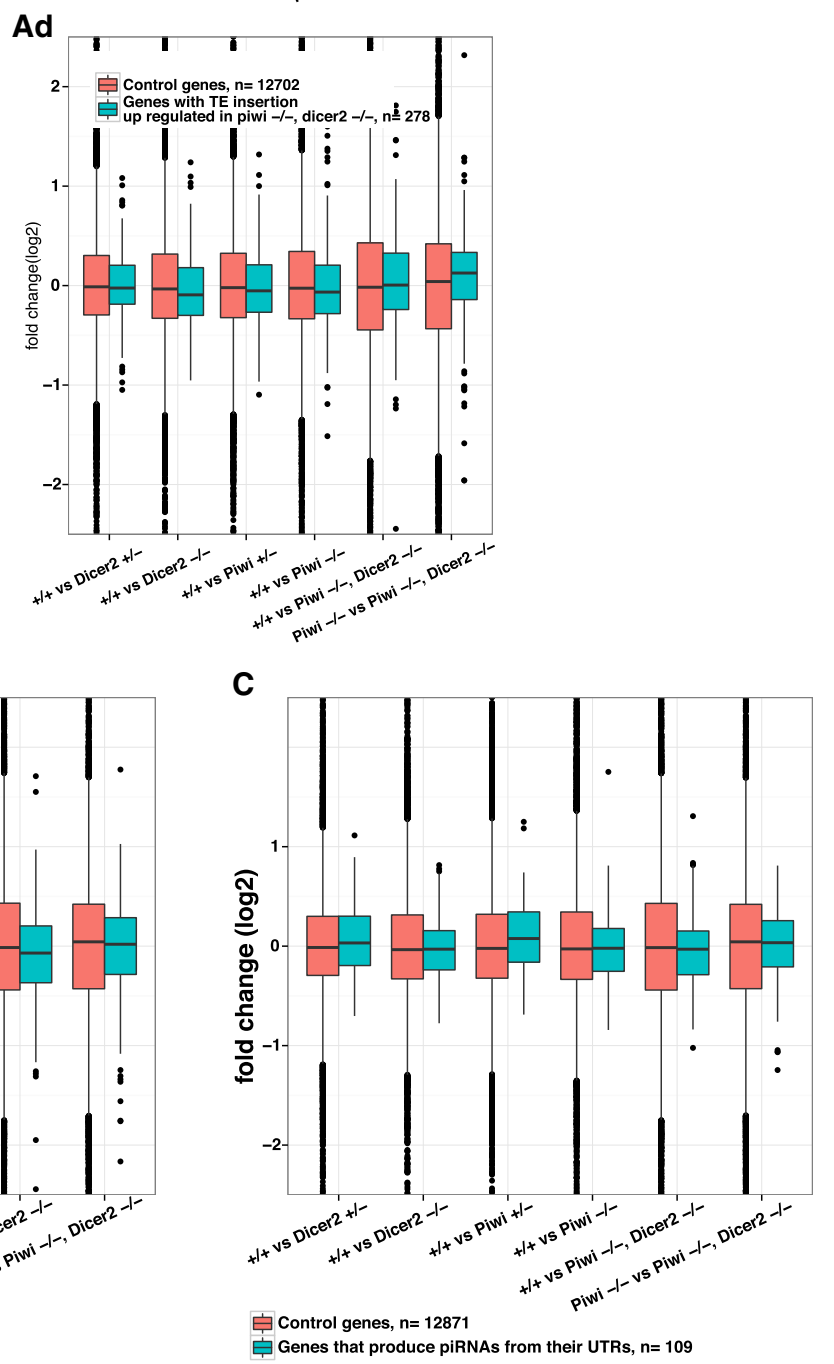

FIGURE 3. Piwi, dicer-2 double mutant in heads does not drastically affect previously reported piwi-regulated genes in gonads. Boxplots of fold change for genes with reference genome TE insertions in their genomic boundaries do not suggest a trend for these genes to become derepressed across any of the tested mutant conditions ( $x$-axis). We compared genes without TE insertions (red boxes) with genes with insertion of TE of any family (blue boxes, Aa), or with TE insertions that are up-regulated in dicer2 homozygous (blue boxes, Ab), piwi homozygous (Ac), or piwi, dicer-2 double homozygous mutants (Ad). (B) Boxplots of the distribution of fold changes for genes with DNA Damage Gene Ontology terms and $(C)$ genes that have been reported to host piRNA production from their UTRs. 
effect on steady state RNA levels of most of the TE families in heads, our work is in line with this model.

\section{Repression of TEs in the absence of siRNAs}

Dicer-2 mutations caused a strong reduction of TE-specific siRNAs. However, this did not result in major changes of TEs' RNA expression for most TE families in adult heads, with the notable exception of 297. This observation is in line with the results of Ghildiyal et al. (2008) who found 297 expression to be strongly increased in heads of dicer-2 mutants, and Xie et al. (2013) who demonstrated increased somatic transposition of 297 in dicer2 mutants. How repression of specific TEs escapes from Piwi and mainly relies on siRNA pathways requires further investigation.

\section{A dual-layer TE repression by small RNAs}

We show that in adult heads, the loss of only piwi or Dicer2 does not lead to significant change in expression of most TE families. However, loss of piwi is associated with increased levels of antisense TE transcripts and of siRNAs, whereas double piwi Dicer-2 mutants show increased levels of transcripts from a large panel of TE families. Similar observations were made recently in ovarian somatic cells where double knockdown of piwi and Dicer-2 leads to synergistic derepression of LTR retrotransposons (Barckmann et al. 2018). We thus propose a dual-layer repression mechanism whereby residual expression of TE that results from incomplete piwi-mediated TGS feeds Dicer-2 for siRNA production that in turn reduces TE transcript levels through PTGS. This mechanism might be especially relevant for TEs that can escape Piwi-mediated repression, such as 297, or TEs that do not induce yet Piwi-mediated TGS because they are not integrated into piRNA clusters. It could also play a role in aging since an increase in TE expression and transposition was observed in aging adult flies, which was amplified in Dicer-2 mutants and mitigated by Dicer-2 overexpression (Wood et al. 2016).
In evolutionary terms, the "failsafe," dual-layer repression mechanism could help during the early steps of a TE invasion of a fly population. Thus, siRNA-mediated PTGS would maintain a tolerable load of the TE transcripts, until the TE has integrated into a piRNA cluster and triggered a stable Piwi-mediated TGS.

\section{MATERIALS AND METHODS}

\section{Fly stocks}

Flies were grown on standard Drosophila food at $25^{\circ} \mathrm{C}$. All flies were brought into the $w^{m 4}$ background (Muller 1930). Dicer$2^{\mathrm{R} 416 \mathrm{X}}$ and dicer-2 ${ }^{\mathrm{L} 811 \mathrm{fsx}}$ alleles were previously described (Lee et al. 2004). Piwi ${ }^{2}$ and piwi ${ }^{3}$ alleles were previously described (Cox et al. 1998). Double mutants were generated by crossing virgin female dcr $2^{\mathrm{R} 416 \mathrm{X}} / \mathrm{CyO}-\mathrm{GFP}$ to male piwi ${ }^{3} / \mathrm{CyO}-\mathrm{GFP}$ flies. Offspring virgin $\mathrm{dcr} 2^{\mathrm{R} 416 \mathrm{X}} / \mathrm{piwi}^{3}$ flies were then crossed to male wm4; $\mathrm{Ln}^{2 \mathrm{R}} \mathrm{Gla}$, wgGla ${ }^{1}, \mathrm{Bc}^{1} / \mathrm{CyO}-\mathrm{GFP}$ to establish wm4; $\mathrm{piwi}^{2}$, dicer-2 ${ }^{\mathrm{R} 416 \mathrm{X}} / \mathrm{CyO}-\mathrm{GFP}$ stocks. Stocks were then screened by $\mathrm{PCR}$ for the presence of the piwi mutation. The same procedure was applied to generate wm4; piwi $^{3}$, dicer- $2^{\text {L811Fsx }} /$ CyO-GFP stocks. Table 1 provides the detailed genotype of all mutant combinations used.

\section{RNA extraction and sequencing}

Head RNAs were prepared as follows. One- to 2-d-old flies were $\mathrm{CO}_{2}$ anesthetized, sorted by sex and genotype, transferred into $15 \mathrm{~mL}$ Falcon tubes, and frozen in liquid nitrogen. The procedure was repeated for multiple days until pools of 50 to 100 flies were obtained per biological replicate. Heads were separated from bodies by vortexing, followed by sieving and a careful selection of heads from thoraces and legs on a cooled metal plate under a binocular loop. Heads were collected into $2 \mathrm{~mL}$ Precellys tubes for hard tissues and covered by $1 \mathrm{ml}$ TRIzol. Heads were homogenized in two rounds of $5000 \mathrm{rpm}$ for $30 \mathrm{sec}$ using a Precellys24 Tissue Homogenizer. Homogenate was centrifuged for $30 \mathrm{sec}$ at 13,000 rpm and supernatant transferred into a new $2 \mathrm{~mL}$ tube, $200 \mu \mathrm{L}$ of chloroform was added and tubes were thoroughly vortexed. Further purification was as in Rio et al. (2010). The remaining DNA was removed using Fermentas DNase I, RNasefree following the manufacturer's instructions.

TABLE 1. Genotype data for samples subjected to RNA sequencing

\begin{tabular}{|c|c|c|c|}
\hline Indicated name & Genotype & Maternal genotype & Paternal genotype \\
\hline$+/+$ & wm4;+/+ & wm4;+/+ & wm $4 ;+/+$ \\
\hline dicer-2 G31R/- & $\mathrm{wm} 4 ; \mathrm{dcr} 2^{\mathrm{G} 31 \mathrm{R}} / \mathrm{dcr} 2^{\mathrm{L} 811 \mathrm{Fsx}}$ & wm4; dcr2 ${ }^{\text {L811Fsx }} /$ CyO-GFP & wm4;dcr2 $2^{\mathrm{G} 31 \mathrm{R}} / \mathrm{CyO}-\mathrm{GFP}$ \\
\hline dicer-2 +/- & $\mathrm{wm} 4 ; \mathrm{dcr} 2^{\mathrm{L811Fsx} /+}$ & wm4;dcr2 2811 Fsx/CyO-GFP & wm4;+/+ \\
\hline dicer-2 -/- & $\mathrm{wm} 4 ; \mathrm{dcr} 2^{\mathrm{L811Fsx}} / \mathrm{dcr} 2^{\mathrm{R} 416 \mathrm{X}}$ & wm4;dcr2 ${ }^{\text {L811Fsx }} /$ CyO-GFP & wm4;dcr2 ${ }^{\mathrm{R} 416 \mathrm{X}} / \mathrm{CyO}$-GFP \\
\hline piwi +/- & wm4;piwi ${ }^{2} /+$ & wm4;piwi²/CyO-GFP & wm $4 ;+/+$ \\
\hline piwi -/- & wm4;piwi ${ }^{2} /$ piwi $^{3}$ & wm4;piwi²/CyO-GFP & wm4;piwi³/CyO-GFP \\
\hline dicer-2 -/-, piwi -/- & $\begin{array}{l}\text { wm4; piwi }{ }^{2} \text {, dicer- } 2^{\text {R416X }} / \text { piwi }^{3} \text {, } \\
\text { dicer-2 } 2^{\text {L811Fsx }}\end{array}$ & wm4;piwi ${ }^{2}$, dicer-2 ${ }^{\mathrm{R} 416 \mathrm{X}} / \mathrm{CyO}$-GFP & wm4;piwi ${ }^{3}$, dicer-2 ${ }^{\text {L811Fsx }} /$ CyO-GFP \\
\hline
\end{tabular}


Small RNA library preparation and sequencing was performed on an Illumina HiSeq 2500 at Fasteris Life Sciences SA (Plan-lesOuates, Switzerland) using the Drosophila small RNA track based on the Illumina TruSeq protocol.

RNA-seq was performed in biological triplicates for $+/+$, piwi $-/+$, piwi $-/-$, dicer-2 -/+, and dicer-2 -/-, with one replicate per condition sequenced in paired-end mode $(2 \times 101)$ and two replicates sequenced in single-read mode $(1 \times 51)$. piwi $-/-$, dicer-2 -/- samples were sequenced in biological duplicates in single-read mode. Total RNA was depleted of rRNA using Ribo-Zero Gold Kit (Epicentre). Directional RNA-seq library preparation and sequencing was performed at the Genomic Paris Centre using the Epicentre ScriptSeq v2 RNA-Seq Library Preparation Kit on an Illlumina HiSeq 2000 instrument.

All sequence files generated in this study are available from the EBI European Nucleotide Archive database (http://www.ebi.ac. uk/ena/) under study accession numbers PRJEB8519 (small RNA-seq GZI-2) and PRJEB25033 (other sequence runs).

\section{Computational analysis}

The complete computational analysis pipeline was run on our inhouse Galaxy server. All necessary workflows and tools will be publicly available at http://mississippi.fr/ (see Figures 1-3 and Supplemental Figures 1 and 2).

All small RNA libraries were quality controlled, sequencing adapter-clipped, and converted to fasta reads. All reads that aligned to ribosomal RNA were discarded. All small RNA alignments were done using bowtie 0.12 .7 and the option "-v 1", which allows one mismatch between sequenced read and reference sequence (Langmead et al. 2009). Note that when using the Bowtie option "-v 1", sequence quality values are not taken into account for alignments. To produce Supplemental Figure 1 , fasta reads were aligned to the Drosophila genome (FlyBase release 5.49) (St. Pierre et al. 2014), randomly placing reads that align equally well in multiple genomic locations (multimapper) using the bowtie option "-M 1". Size distribution and ping-pong signature were calculated using the mississippi toolsuite (https:// testtoolshed.g2.bx.psu.edu/view/drosofff/mississipi_toolsuite_ beta). The ping-pong signature was calculated by counting the number of pairs that overlapped between 5 and 15 nt between sense and antisense aligned reads and transforming the obtained counts into z-scores (each count subtracted by the mean and divided by the standard deviation). Ping-pong positive libraries were selected by having a z-score higher than 2 for a $10 \mathrm{bp}$ overlap and more than 20 pairs overlapping by $10 \mathrm{nt}$. Pingpong negative libraries were selected by having a negative $z$ score. To obtain a list of differentially expressed miRNAs between ping-pong positive and ping-pong negative libraries, reads were matched to the Drosophila pre-miRNAs of the miRBase 20 release (Griffiths-Jones 2004; Griffiths-Jones et al. 2006, 2008; Kozomara and Griffiths-Jones 2011, 2014). Differential expression profiling between ping-pong positive and ping-pong negative libraries was performed using edgeR_3.8.2 (Robinson et al. 2010; McCarthy et al. 2012) with standard settings. For simulating contamination with testis RNA two testis-libraries (accessions SRX135547, SRX023726) were downsampled to 10 million reads and pooled, and 50,000 randomly selected reads were added to $2.45 \times 10^{6}$ randomly selected reads from ping-pong negative libraries. piRNA signature was calculated as before. Differential miRNA expression was calculated between simulated libraries and ping-pong negative libraries of equal size (randomly downsampled to $2.5 \times 10^{6}$ ), with libraries that were sampled from the same initial ping-pong negative library paired as a blocking factor. This allows for obtaining an accurate list of miRNAs (contamination signature) that should be expected to be significantly changed in abundance if a contamination occurred. Size distribution for small RNAs that align to TEs (Fig. 1A,B) was calculated from reads that matched any of the canonical TE sequences with one mismatch allowed, excluding reads that matched to ribosomal RNA, tRNA or abundant insect viruses. Abundance of $21 \mathrm{nt}$ antisense RNA for each TE family was calculated by filtering reads to $21 \mathrm{nt}$ length and aligning reads to canonical TE sequences, allowing only unique reads using the bowtie option "-v 1 ". Only antisense reads were counted, and only TEs with on average 20 reads per library were analyzed. Between-library normalized $21 \mathrm{nt}$ antisense TE counts were obtained by pooling these with miRNA reads (obtained as before) and calculating a normalization factor using the DESeq (Anders and Huber 2010) function estimateSizeFactors. $\log _{2}$ fold changes were calculated by dividing normalized reads of mutants by normalized reads of controls and taking the logarithm. Differences of the population of $\log _{2}$ fold changes were tested using a two-tailed Mann-Whitney $U$ test. To calculate mismatch frequencies for $21 \mathrm{nt}$ small RNA, ribosomal, noncoding RNA and viral reads were filtered out. Remaining reads were aligned to the collection of TE insertions (FlyBase version 5.49), allowing one mismatch. Each possible mismatch was counted and divided by the total number of $21 \mathrm{nt}$ reads aligned to the collection of TE insertions.

For gene expression profiling, reads were quality filtered using the FASTX toolkit with a Quality cutoff of 30 for $90 \%$ of the read. For the paired-end libraries, only the R1 read of the pair was used and trimmed to $51 \mathrm{nt}$ to avoid differences in read alignments between sequencing runs. Reads were then aligned to the Drosophila genome release $5(\mathrm{dm} 3)$ using TopHat2 (Kim et al. 2013). Default parameters were used, except that we supplied Gene Model annotations from the UCSC Genome Browser for dm3 (http://support.illumina.com/sequencing/sequencing_ software/igenome.html). Read counting was performed using featureCounts (Liao et al. 2014) guided by the aforementioned Gene Model file.

For TE expression profiling, reads were further trimmed to $30 \mathrm{nt}$ and aligned to canonical TEs using bowtie 0.12.7, allowing two mismatches and only uniquely matching reads. Sense and antisense reads were counted and merged with gene counts. Differential expression profiling was performed using edgeR (Robinson et al. 2010; McCarthy et al. 2012). Genes with less than five reads on average across libraries were discarded from the analysis. Diverging from the default, we used Full Quantile between-library normalization as implemented by the EDAseq package (Risso et al. 2011) and removed unwanted variation using replicate samples with the RUVs function (choosing $k=2$ ) implemented in the RUVseq package (Risso et al. 2014). Library sequencing method (paired-end versus single-end) was introduced together with gene-wise Full Quantile normalization offsets and gene-wise RUV offsets as covariates in the edgeR design formula. All libraries were tested for differential gene expression against the wild-type, and in addition the double mutant was also tested against the piwi -/- mutant. Proportional Venn 
diagrams in Figure 2C,D were drawn using the Vennerable package (https://github.com/js229/Vennerable). The Spearman rank correlation and corresponding $P$-value between $\log _{2}$ fold changes in TE 21 nt antisense RNA ( $\log _{2}$ fold change calculated from data underlying Fig. 1C) and sense TE transcript expression was calculated with the rcorr function in the Hmisc R package (http://cran.rproject.org/web/packages/Hmisc/). All graphs were plotted using ggplot2 (http://ggplot2.org/). GO terms for DNA damage and genes with TE insertions were retrieved from FlyBase (St. Pierre et al. 2014).

\section{SUPPLEMENTAL MATERIAL}

Supplemental material is available for this article.

\section{ACKNOWLEDGMENTS}

We thank S. Chambeyron and A. Pelisson for sharing unpublished data and the Galaxy community for their support. This work received financial support to C.A. from the Agence Nationale de la Recherche grants ANR-13-BSV2-0007 and ANR-10-BLAN1210 and to C.C. and C.A. from the IBPS grant "Action Initiative 2018." M.v.d.B. had a PhD fellowship from the Ministère de la Recherche et de l'Enseignement Supérieur. The École Normale Supérieure genomic core facility was supported by the France Génomique national infrastructure, funded as part of the "Investissements d'Avenir" program managed by the Agence Nationale de la Recherche (contract ANR-10-INBS-09).

Author contributions: M.v.d.B. performed the experiments and the computational analyses and prepared figures and tables. J.P. and M.e.a.A.C. performed the transcriptome sequencing experiments. B.d.S. and C.C. performed genetic crosses. C.A. conceived the project. M.v.d.B., C.C. and C.A. wrote the manuscript.

Received May 3, 2018; accepted September 5, 2018.

\section{REFERENCES}

Anders S, Huber W. 2010. Differential expression analysis for sequence count data. Genome Biol 11: R106.

Barckmann B, El-Barouk M, Pélisson A, Mugat B, Li B, Franckhauser C, Fiston Lavier A-S, Mirouze M, Fablet M, Chambeyron S. 2018. The somatic piRNA pathway controls germline transposition over generations. Nucleic Acids Res. doi:10.1093/nar/gky761.

Brennecke J, Aravin AA, Stark A, Dus M, Kellis M, Sachidanandam R, Hannon GJ. 2007. Discrete small RNA-generating loci as master regulators of transposon activity in Drosophila. Cell 128: 1089-1103.

Brower-Toland B, Findley SD, Jiang L, Liu L, Yin H, Dus M, Zhou P, Elgin SCR, Lin H. 2007. Drosophila PIWI associates with chromatin and interacts directly with HP1a. Genes Dev 21: 2300-2311.

Cenik ES, Fukunaga R, Lu G, Dutcher R, Wang Y, Tanaka Hall TM, Zamore PD. 2011. Phosphate and R2D2 restrict the substrate specificity of Dicer-2, an ATP-driven ribonuclease. Mol Cell 42: 172-184.

Cox DN, Chao A, Baker J, Chang L, Qiao D, Lin H. 1998. A novel class of evolutionarily conserved genes defined by piwi are essential for stem cell self-renewal. Genes Dev 12: 3715-3727.

Czech B, Malone CD, Zhou R, Stark A, Schlingeheyde C, Dus M, Perrimon N, Kellis M, Wohlschlegel JA, Sachidanandam R, et al. 2008. An endogenous small interfering RNA pathway in Drosophila. Nature 453: 798-802.

Ghildiyal M, Seitz H, Horwich MD, Li C, Du T, Lee S, Xu J, Kittler ELW, Zapp ML, Weng Z, et al. 2008. Endogenous siRNAs derived from transposons and mRNAs in Drosophila somatic cells. Science 320: 1077-1081.

Griffiths-Jones S. 2004. The microRNA registry. Nucleic Acids Res 32: D109-D111.

Griffiths-Jones S, Grocock RJ, van Dongen S, Bateman A, Enright AJ. 2006. miRBase: microRNA sequences, targets and gene nomenclature. Nucleic Acids Res 34: D140-D144.

Griffiths-Jones S, Saini HK, van Dongen S, Enright AJ. 2008. miRBase: tools for microRNA genomics. Nucleic Acids Res 36: D154-D158.

Gu T, Elgin SCR. 2013. Maternal depletion of Piwi, a component of the RNAi system, impacts heterochromatin formation in Drosophila. PLoS Genet 9: e1003780.

Gunawardane LS, Saito K, Nishida KM, Miyoshi K, Kawamura Y, Nagami T, Siomi H, Siomi MC. 2007. A slicer-mediated mechanism for repeat-associated siRNA $5^{\prime}$ end formation in Drosophila. Science 315: 1587-1590.

Jin Z, Flynt AS, Lai EC. 2013. Drosophila piwi mutants exhibit germline stem cell tumors that are sustained by elevated Dpp signaling. Curr Biol 23: 1442-1448.

Keegan LP, Brindle J, Gallo A, Leroy A, Reenan RA, O'Connell MA. 2005. Tuning of RNA editing by ADAR is required in Drosophila. EMBO J 24: 2183-2193.

Kim D, Pertea G, Trapnell C, Pimentel H, Kelley R, Salzberg SL. 2013. TopHat2: accurate alignment of transcriptomes in the presence of insertions, deletions and gene fusions. Genome Biol 14: R36.

Kozomara A, Griffiths-Jones S. 2011. miRBase: integrating microRNA annotation and deep-sequencing data. Nucleic Acids Res 39: D152-D157.

Kozomara A, Griffiths-Jones S. 2014. miRBase: annotating high confidence microRNAs using deep sequencing data. Nucleic Acids Res 42: D68-D73.

Langmead B, Trapnell C, Pop M, Salzberg SL. 2009. Ultrafast and memory-efficient alignment of short DNA sequences to the human genome. Genome Biol 10: R25.

Lau NC, Robine N, Martin R, Chung W-J, Niki Y, Berezikov E, Lai EC. 2009. Abundant primary piRNAs, endo-siRNAs, and microRNAs in a Drosophila ovary cell line. Genome Res 19: 1776-1785.

Le Thomas A, Rogers AK, Webster A, Marinov GK, Liao SE, Perkins EM, Hur JK, Aravin AA, Tóth KF. 2013. Piwi induces piRNA-guided transcriptional silencing and establishment of a repressive chromatin state. Genes Dev 27: 390-399.

Lee YS, Nakahara K, Pham JW, Kim K, He Z, Sontheimer EJ, Carthew RW. 2004. Distinct roles for Drosophila Dicer-1 and Dicer-2 in the siRNA/miRNA silencing pathways. Cell 117: 69-81.

Lewis SH, Quarles KA, Yang Y, Tanguy M, Frézal L, Smith SA, Sharma PP, Cordaux R, Gilbert C, Giraud I, et al. 2018. Pan-arthropod analysis reveals somatic piRNAs as an ancestral defence against transposable elements. Nat Ecol Evol 2: 174-181.

Li W, Prazak L, Chatterjee N, Grüninger S, Krug L, Theodorou D, Dubnau J. 2013. Activation of transposable elements during aging and neuronal decline in Drosophila. Nat Neurosci 16: 529-531.

Liao Y, Smyth GK, Shi W. 2014. featureCounts: an efficient general purpose program for assigning sequence reads to genomic features. Bioinformatics 30: 923-930.

Malone CD, Brennecke J, Dus M, Stark A, McCombie WR, Sachidanandam R, Hannon GJ. 2009. Specialized piRNA pathways act in germline and somatic tissues of the Drosophila ovary. Cell 137: 522-535.

Mani SR, Megosh H, Lin H. 2014. PIWI proteins are essential for early Drosophila embryogenesis. Dev Biol 385: 340-349. 
McCarthy DJ, Chen Y, Smyth GK. 2012. Differential expression analysis of multifactor RNA-seq experiments with respect to biological variation. Nucleic Acids Res 40: 4288-4297.

Mirkovic-Hösle M, Förstemann K. 2014. Transposon defense by endosiRNAs, piRNAs and somatic pilRNAs in Drosophila: contributions of Loqs-PD and R2D2. PLoS One 9: e84994.

Muller HJ. 1930. Types of visible variations induced by $X$-rays in Drosophila. J Genet 22: 299-334.

Nagao A, Mituyama T, Huang H, Chen D, Siomi MC, Siomi H. 2010. Biogenesis pathways of piRNAs loaded onto $A G O 3$ in the Drosophila testis. RNA 16: 2503-2515.

Pal-Bhadra M, Leibovitch BA, Gandhi SG, Rao M, Bhadra U, Birchler JA, Elgin SCR. 2004. Heterochromatic silencing and HP1 localization in Drosophila are dependent on the RNAi machinery. Science 303: 669-672.

Palladino MJ, Keegan LP, O'Connell MA, Reenan RA. 2000. dADAR, a Drosophila double-stranded RNA-specific adenosine deaminase is highly developmentally regulated and is itself a target for RNA editing. RNA 6: 1004-1018.

Perrat PN, DasGupta S, Wang J, Theurkauf W, Weng Z, Rosbash M, Waddell S. 2013. Transposition-driven genomic heterogeneity in the Drosophila brain. Science 340: 91-95.

Rangan P, Malone CD, Navarro C, Newbold SP, Hayes PS, Sachidanandam R, Hannon GJ, Lehmann R. 2011. piRNA production requires heterochromatin formation in Drosophila. Curr Biol 21: 1373-1379.

Reinhardt A, Feuillette S, Cassar M, Callens C, Thomassin H, Birman S, Lecourtois M, Antoniewski C, Tricoire H. 2012. Lack of miRNA misregulation at early pathological stages in Drosophila neurodegenerative disease models. Front Genet 3: 226.

Rio DC, Ares M Jr, Hannon GJ, Nilsen TW. 2010. Purification of RNA using TRIzol (TRI reagent). Cold Spring Harb Protoc 2010: pdb. prot 5439.

Risso D, Schwartz K, Sherlock G, Dudoit S. 2011. GC-content normalization for RNA-seq data. BMC Bioinformatics 12: 480.

Risso D, Ngai J, Speed TP, Dudoit S. 2014. Normalization of RNA-seq data using factor analysis of control genes or samples. Nat Biotechnol 32: 896-902.

Robine N, Lau NC, Balla S, Jin Z, Okamura K, Kuramochi-Miyagawa S, Blower MD, Lai EC. 2009. A broadly conserved pathway generates 3'UTR-directed primary piRNAs. Curr Biol 19: 2066-2076.

Robinson MD, McCarthy DJ, Smyth GK. 2010. edgeR: a Bioconductor package for differential expression analysis of digital gene expression data. Bioinformatics 26: 139-140.

Rouget C, Papin C, Boureux A, Meunier A-C, Franco B, Robine N, Lai EC, Pelisson A, Simonelig M. 2010. Maternal mRNA deadenylation and decay by the piRNA pathway in the early Drosophila embryo. Nature 467: 1128-1132.

Rozhkov NV, Aravin AA, Zelentsova ES, Schostak NG, Sachidanandam R, McCombie WR, Hannon GJ, Evgen'ev MB. 2010. Small RNA-based silencing strategies for transposons in the process of invading Drosophila species. RNA 16: 1634-1645.
Rozhkov NV, Hammell M, Hannon GJ. 2013. Multiple roles for Piwi in silencing Drosophila transposons. Genes Dev 27: 400-412.

Saito K, Inagaki S, Mituyama T, Kawamura Y, Ono Y, Sakota E, Kotani H, Asai K, Siomi H, Siomi MC. 2009. A regulatory circuit for piwi by the large Maf gene traffic jam in Drosophila. Nature 461: 1296-1299.

Sienski G, Dönertas D, Brennecke J. 2012. Transcriptional silencing of transposons by Piwi and maelstrom and its impact on chromatin state and gene expression. Cell 151: 964-980.

Sienski G, Batki J, Senti K-A, Dönertas D, Tirian L, Meixner K, Brennecke J. 2015. Silencio/CG9754 connects the Piwi-piRNA complex to the cellular heterochromatin machinery. Genes Dev 29: 2258-2271.

St. Pierre SE, Ponting L, Stefancsik R, McQuilton P, FlyBase Consortium. 2014. FlyBase 102-advanced approaches to interrogating FlyBase. Nucleic Acids Res 42: D780-D788.

Toledano H, D'Alterio C, Czech B, Levine E, Jones DL. 2012. The let7-Imp axis regulates ageing of the Drosophila testis stem-cell niche. Nature 485: 605-610.

Tubio JMC, Li Y, Ju YS, Martincorena I, Cooke SL, Tojo M, Gundem G, Pipinikas CP, Zamora J, Raine K, et al. 2014. Extensive transduction of nonrepetitive DNA mediated by L1 retrotransposition in cancer genomes. Science 345: 1251343.

Vagin W, Sigova A, Li C, Seitz H, Gvozdev V, Zamore PD. 2006. A distinct small RNA pathway silences selfish genetic elements in the germline. Science 313: 320-324.

Wang SH, Elgin SCR. 2011. Drosophila Piwi functions downstream of piRNA production mediating a chromatin-based transposon silencing mechanism in female germ line. Proc Natl Acad Sci 108: 21164-21169.

Welker NC, Maity TS, Ye X, Aruscavage PJ, Krauchuk AA, Liu Q, Bass BL. 2011. Dicer's helicase domain discriminates dsRNA termini to promote an altered reaction mode. Mol Cell 41: 589-599.

Wen J, Duan H, Bejarano F, Okamura K, Fabian L, Brill JA, Bortolamiol-Becet D, Martin R, Ruby JG, Lai EC. 2015. Adaptive regulation of testis gene expression and control of male fertility by the Drosophila hairpin RNA pathway. [Corrected]. Mol Cell 57: 165-178.

Wood JG, Jones BC, Jiang N, Chang C, Hosier S, Wickremesinghe P, Garcia M, Hartnett DA, Burhenn L, Neretti N, et al. 2016. Chromatin-modifying genetic interventions suppress age-associated transposable element activation and extend life span in Drosophila. Proc Natl Acad Sci 113: 11277-11282.

Wu D, Lamm AT, Fire AZ. 2011. Competition between ADAR and RNAi pathways for an extensive class of RNA targets. Nat Struct Mol Biol 18: 1094-1101.

Xie W, Donohue RC, Birchler JA. 2013. Quantitatively increased somatic transposition of transposable elements in Drosophila strains compromised for RNAi. PLoS One 8: e72163.

Yan Z, Hu HY, Jiang X, Maierhofer V, Neb E, He L, Hu Y, Hu H, Li N, Chen W, et al. 2011. Widespread expression of piRNA-like molecules in somatic tissues. Nucleic Acids Res 39: 6596-6607. 

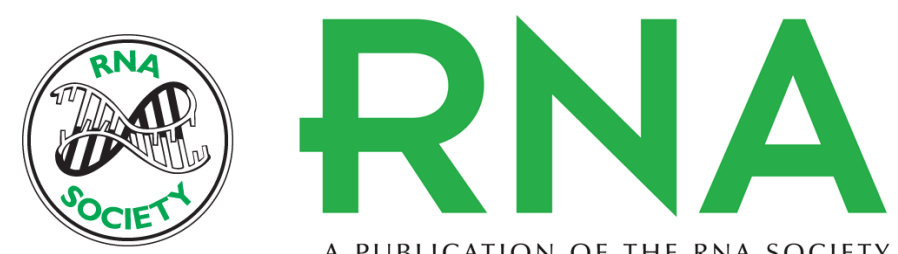

A PUBLICATION OF THE RNA SOCIETY

\section{Dual-layer transposon repression in heads of Drosophila melanogaster}

Marius van den Beek, Bruno da Silva, Juliette Pouch, et al.

RNA 2018 24: 1749-1760 originally published online September 14, 2018

Access the most recent version at doi:10.1261/rna.067173.118

\section{Supplemental http://rnajournal.cshlp.org/content/suppl/2018/09/14/rna.067173.118.DC1 Material}

References This article cites 58 articles, 18 of which can be accessed free at: http://rnajournal.cshlp.org/content/24/12/1749.full.html\#ref-list-1

Open Access Freely available online through the RNA Open Access option.

Creative This article, published in RNA, is available under a Creative Commons License Commons (Attribution 4.0 International), as described at

License http://creativecommons.org/licenses/by/4.0/.

Email Alerting Receive free email alerts when new articles cite this article - sign up in the box at the Service top right corner of the article or click here. 